Meta

Journal des traducteurs

Translators' Journal

\title{
Une traduction (im)possible
}

La version espagnole du Sonnet en X par Octavio Paz

\section{Jean-Claude Masson}

Volume 31, numéro 3, septembre 1986

Prismes de traductions littéraires

Facets of Literary Translation

URI : https://id.erudit.org/iderudit/003594ar

DOI : https://doi.org/10.7202/003594ar

Aller au sommaire du numéro

Éditeur(s)

Les Presses de l'Université de Montréal

ISSN

0026-0452 (imprimé)

1492-1421 (numérique)

Découvrir la revue

Citer cet article

Masson, J.-C. (1986). Une traduction (im)possible : la version espagnole du Sonnet en X par Octavio Paz. Meta, 31(3), 314-320.

https://doi.org/10.7202/003594ar d'utilisation que vous pouvez consulter en ligne.

https://apropos.erudit.org/fr/usagers/politique-dutilisation/ 


\title{
UNE TRADUCTION (IM)POSSIBLE : LA VERSION ESPAGNOLE DU SONNET EN X PAR OCTAVIO PAZ
}

\author{
JEAN-ClaUde MASSON \\ BRUXELLES, BELGIQUE
}

En 1968, peu avant son retour des Indes, Octavio Paz achevait la traduction du Sonnet en $X$ de Mallarmé. Il accompagnait celle-ci d'un commentaire magistral sur le poème, ainsi que de remarques sur sa propre traduction. L'ensemble de ces notes fut publié dans la deuxième section de El Signo y el Garabato, section intitulée précisément : "Teoría y práctica de la traducción" (Mexico, 1973).

$\mathrm{Si}$ j'ai choisi de vous présenter cette traduction et ce commentaire - fortement résumé - c'est pour deux raisons : d'une part, le Sonnet en $X$ semble présenter tous les symptômes de l'intraduisibilité, cumuler les difficultés qui contraignent souvent à recourir aux notes, cette " maladie honteuse " du traducteur ; d'autre part, il m'a paru intéressant d'élaborer un exposé sur la traduction espagnole d'un poème français à partir de la traduction française d'un commentaire en espagnol.

Mais commençons par lire (relire) ce poème de Mallarmé qui ravit ou irrita tant de ses contemporains, ainsi que la version espagnole.

\section{EL SONETO EN IX (1868)}

Ses purs ongles très haut dédiant leur onyx, L'Angoisse, ce minuit, soutient, lampadophore, Maint rêve vespéral brûlé par le Phénix Que ne recueille pas de cinéraire amphore

Sur les crédences, au salon vide : nul ptyx, Aboli bibelot d'inanité sonore,

(Car le Maître est allé puiser des pleurs au Styx Avec ce seul objet dont le Néant s'honore).

Mais proche la croisée au nord vacante, un or Agonise selon peut-être le décor

Des licornes ruant du feu contre une nixe,

Elle, défunte nue en le miroir, encor

Que, dans l'oubli fermé par le cadre, se fixe

De scintillations sitôt le septuor.

Stéphane Mallarmé

TRADUCCIÓN (1968)

A Tomás Segovia

El de sus puras uñas ónix, alto en ofrenda,

La Angustia, es medianoche, levanta, lampadóforo, Mucho vesperal sueño quemado por el Fénix

Que ninguna recoge ánfora cineraria : 
Sala sin nadie ni en las credencias conca alguna, Espiral espirada de inanidad sonora,

(El Maestro se ha ido, llanto en la Estigia capta Con ese solo objeto nobleza de la Nada.)

Mas cerca la ventana vacante al norte, un oro Agoniza según tal vez rijosa fábula

$X$ De ninfa alanceada por llamas de unicornios

Y ella apenas difunta desnuda en el espejo

Que ya en las nulidades que clausura el marco

Del centellar se fija súbito el septimino.

Octavio Paz

\section{VERSIFICATION ET SYNTAXE}

La première chose qui frappe à la lecture de cette version espagnole est l'absence des rimes en $\mathrm{X}$, une des principales difficultés du poème. Nous voyons qu'Octavio Paz ne s'en préoccupe guère, persuadé que l'on peut (re)créer un équivalent du sonnet ${ }^{1}$ tout en délaissant le problème de ces rimes abstruses 2 . Il pensait pourtant à cette époque refaire un jour une version avec des rimes présentant les mêmes difficultés et sonorités (il m'a déclaré dernièrement qu'il n'avait pas encore eu l'occasion de retravailler à sa traduction). Pour ce qui est du rythme, Paz essaie de suivre celui de l'alexandrin en tempérant au maximum le caractère sculptural de l'endécasyllabe espagnol. Enfin, pour restituer la syntaxe torturée de Mallarmé, il s'inspire des baroques espagnols. C'est le cas, par exemple, au premier vers, où il éloigne l'article de son substantif, ou au vers quatre, en séparant ninguna du mot ánfora auquel il se rapporte, s'inspirant ici d'un vers de Góngora : "en una de fregar cayó caldera ". Et ce n'est pas un hasard si Paz dédie sa traduction à Tomás Segovia, un des plus grands spécialistes de la métrique espagnole.

\section{STRUCTURATION}

Ce sonnet est composé de deux phrases, alors que le schéma traditionnel du genre, depuis Pétrarque, présente quatre phrases : un sonnet quasi syllogistique. Mallarmé, lui, revient au dualisme néo-platonicien qui est consubstantiel à l'apparition du sonnet.

Dans le sonnet classique, le premier quatrain est une exposition, le second sa négation ou son altération, le premier tercet est la crise et le second le dénouement. Le sonnet consiste en une proposition, ou plutôt, en quatre propositions enchaînées par une logique non moins rigoureuse que celle unissant les membres d'un syllogisme. Sans altérer cette structure logico-poétique, Mallarmé atténue l'opposition entre quatrains d'une part, entre tercets d'autre part. Le schéma du sonnet traditionnel pourrait se représenter ainsi ( $a$ et a' désignant les quatrains, b et b' les tercets) :

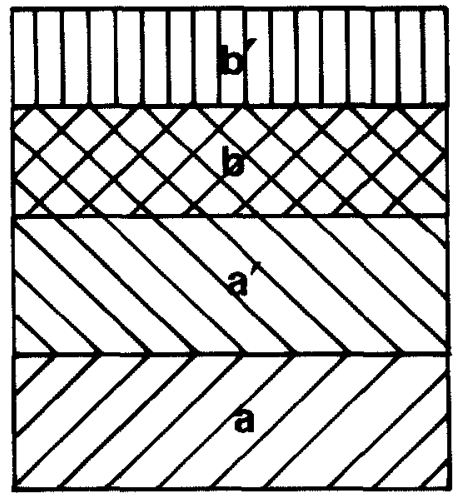


Par contre, la représentation graphique du Sonnet en $X$ serait :

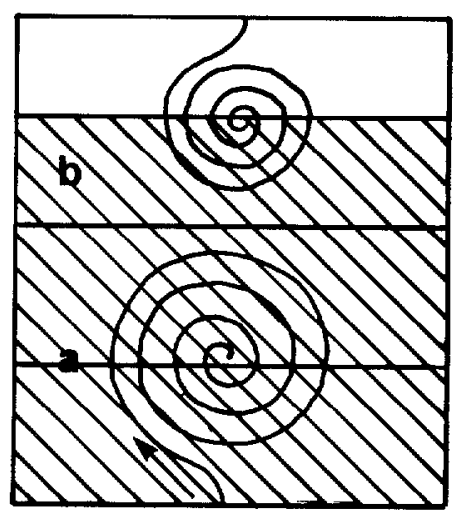

La première phrase s'enroule comme une spirale qui se replie jusqu'à s'annuler ; la seconde se déplie jusqu'à se confondre avec l'univers - et se dissiper.

\begin{abstract}
A) Première ph(r)ase
Au premier vers du premier quatrain, "très haut dédiant " (notons que Paz traduit par " offrande", terme plus fort que "dédiant ") souligne d'entrée de jeu l'aspect "rituel " du poème, son caractère théâtral, dramatique. Le second vers y fait écho avec le mot "lampadophore" (porte-flambeau utilisé lors des cérémonies religieuses dans l'Antiquité). En outre, la traduction de "ce minuit " par es medianoche (il est minuit) actualise davantage la suspension du temps ${ }^{3}$, fixité temporelle qui caractérise aussi les "moments forts" des pratiques religieuses, telle l'Élévation dans le rite catholique. Ici, dans ce premier quatrain, l'angoisse lève les bras et soutient tous les rêves du crépuscule, rêves de consommation et de résurrection qui furent brûlés par le soleil - Phénix. L'angoisse (" héros " du premier quatrain) est une métaphore de minuit : fin d'une journée et début d'une autre. Heure angoissante car, en elle, l'unité du temps et sa continuité semblent se briser : le soleil ressuscitera-t-il de ses cendres, ou bien cette heure marque-t-elle le commencement d'une obscurité hors du temps? En dépit des certitudes successives que lui ont apporté la magie, la religion et la science, l'homme se répète cette question depuis son apparition sur la terre. Dans le noir total de ce premier quatrain, noir impersonnel et cosmique, l'unique refiet est celui de l'onyx des ongles de l'angoisse (entre parenthèses : onyx vient de ovux : ongle). Ici donc, Mallarmé nous présente un aspect, le dernier, de ce qu'il appelle la "tragédie de la nature" dans une note à sa traduction du traité de mythologie de George Cox (les Dieux antiques) où il écrit :
\end{abstract}

... le changement des Saisons, la naissance de la Nature au printemps, sa plénitude estivale de vie et sa mort en automne, enfin sa disparition totale pendant l'hiver (phases qui correspondent au lever, à midi, au coucher, à la nuit) [sont] le grand et perpétuel sujet de la $\mathrm{My}$ thologie : la double évolution solaire, quotidienne et annuelle. (...) la Tragédie de la Nature.

Ainsi, l'angoisse n'est pas psychologique : c'est une phase du rituel solaire, rituel dont est encore connotée l'" amphore cinéraire » du quatrième vers. Au moyen de successives réductions analogiques, Mallarmé condense en ce premier quatrain les aspects nocturnes et négatifs du drame de la nature : solstice d'hiver — minuit - angoisse. 
Dans le second quatrain, la principale difficulté sémantique est le mot "ptyx ", créé - en tant que nom commun - par Mallarmé. Celui-ci, en effet, confiait dans une lettre datée du 3 mai 1868 et adressée à son ami Eugène Lefébure :

... je n'ai que trois rimes en $i x$, concertez-vous pour m'envoyer le sens réel du mot ptyx : on m'assure qu'il n'existe dans aucune langue, ce que je préférerais de beaucoup à fin de me donner le charme de le créer par la magie de la rime.

Nous voici donc, semble-t-il, face à une sorte d'hapax parfaitement intraduisible. Pour trouver un équivalent à ce mot, $\mathrm{Paz}$ s'en remet à l'analyse de la critique belge Émilie Noulet, analyse avalisée par la majorité des critiques :

... pour lui donner un sens, il faut remonter à son origine grecque où l'idée de pli est fondamentale... Le contexte aidant, on peut en déduire que "ptyx " désigne une conque, un de ces coquillages qui, collé à l'oreille, fait entendre le bruit de la mer ${ }^{4}$.

Ainsi, l'étude de l'œuvre entière du poète (tâche primordiale du traducteur aux yeux de Steiner et de tant d'autres), ainsi que l'interprétation de cette œuvre (par les critiques et le traducteur lui-même) permet à Octavio Paz de surmonter la difficulté essentielle présentée par le mot "ptyx » et de traduire par un équivalent : conca (conque, coquillage). Nous verrons plus loin que l'image de cette conque marine s'avère capitale pour la compréhension du texte.

Au vu de la ponctuation, le dernier vers du premier quatrain doit se lire en considérant "Sur les crédences" (premier vers du second quatrain) comme un enjambement ; le sens est donc : " que ne recueille (aucune) amphore cinéraire (parmi celles qui reposent) sur les crédences, dans la salle vide : aucun ptyx". Étant donné que "nul ptyx » est apposé à " amphore " et, de plus, est son homologue, Paz a choisi de séparer les quatrains par deux points. Ainsi modifie-t-il légèrement le sens puisque ici, ce qui est sur les crédences, ce ne sont pas les amphores (inexistantes), mais le coquillage (non moins inexistant). Ce faisant, le traducteur ne rompt nullement l'unité de la phrase.

Revenons, à présent, à l'interprétation. Dans le second quatrain, nous passons du monde naturel au monde humain. Absence à nouveau : celle du maître de maison, qui est en même temps le poète, l'initié et le pénitent. Absence, aussi bien, de cet objet énigmatique "dont le Néant s'honore".

"Le Maître est allé puiser des pleurs au Styx " $:$ la descente au Styx présente une double signification. D'une part, il s'agit d'une descente au royaume souterrain, d'une initiation requérant la mort symbolique du néophyte et sa résurrection (comme celle du poète par l'écriture); d'autre part, la descente au Styx représente un renoncement au moi, une épochè : la conscience se retire de soi-même, se vide et devient transparence impersonnelle. Ainsi, l'analogie qui unissait minuit à l'angoisse unit le maitre à la chambre vide. Le maitre est une métaphore du Néant : sa conscience de soi n'est que le savoir de son absence de soi. Transformations analogiques : la chambre = le maître (sa conscience vide) $=$ le Néant. Il en va de même de l'outil du poète, "Aboli bibelot d'inanité sonore", et c'est ici que l'image de la conque prend toute sa signification. C'est une structure qui se replie sur elle-même. D'après Jean-Pierre Richard (l'Univers imaginaire de Mallarmé, 1961), le pli est une forme vitale de la réflexion : penser, c'est " se replier ". Mais le pli est aussi charnel : symbole réflexif et érotique, le coquillage est en même temps une chambre, une maison. En outre, il enferme la mer et ainsi, c'est un emblème de la vie universelle, de sa mort et de sa renaissance perpétuelles. En même temps, il ne contient rien : du vent. Cette dualité fait du coquillage un quelconque bibelot en même temps qu'un objet rituel. Parfaitement double, le coquillage résume, dans son immense petitesse, toutes les autres images, métaphore de métaphores : solstice d'hiver $=$ minuit 
$=$ angoisse $($ universelle $)=$ chambre vide $=$ Néant $=$ maître (conscience en $"$ creux $")$ $=$ conque (bibelot aboli). Mais la séquence est réversible si le mouvement de repli est suivi d'un mouvement de dépliement : conque (objet rituel) $=$ Musique $=$ Héros (poète) $=$ Théâtre (dialogue, communion) $=$ conscience universelle $=$ midi $=$ solstice d'été. Le coquillage est le point de convergence de toutes les lignes de force et le lieu de leur métamorphose. Lui-même est métamorphose. Une fois encore, c'est l'ensemble de cette analyse qui permet à Octavio Paz de trouver un équivalent à " aboli bibelot 》 : espiral espirada. Espirar signifie à la fois aspirer et expirer : emblème de la mer, de la musique (la conque est aussi un instrument à vent), du va-et-vient de la vie humaine.

Con ese solo objeto nobleza de la Nada (Avec ce seul objet noblesse du Néant) : remarquons les allitérations, les rimes intérieures, ainsi que la traduction - magistrale par simple apposition de termes. (Dans son œuvre entière, que ce soit pour définir ou expliciter, Octavio Paz préfêre ces simples juxtapositions aux conjonctions, aux " mots chevilles ».)

\section{B) Seconde ph(r)ase}

La conclusion des quatrains est négative : obscurité, absence. Cependant, dans le cadre doré du miroir, une lumière (" un or ") agonise ${ }^{6}$. Et les spasmes de cette agonie reproduisent les mouvements d'une scène mythologique peinte ou gravée dans l'encadrement d'un miroir : un groupe de licornes attaque une nixe (nymphe dans la mythologie germanique). Nouvelle analogie, cette fois entre les râles de l'agonie et la violence érotique et incendiaire. Le premier tercet répète au niveau de la fantaisie individuelle - l'adverbe de doute indique qu'il s'agit peut-être d'une sensation visuelle - et à celui de l'imagination mythique - les unicornes et la nixe - le thème des deux quatrains le crime de la nuit, la mort du soleil. La relation unissant le premier tercet au premier quatrain est très étroite : le fil de lumière du cadre et l'onyx des ongles de l'angoisse ; la mort par le feu des songes vespéraux tout comme de la nymphe. Dans le premier cas, le soleil, héros viril, s'incendie lui-même : c'est le Phénix ; dans le second, transformé en licorne, il embrase l'objet désiré : la nymphe. Les deux quatrains et le premier tercet sont trois représentations de ce que Mallarmé appelait "La Tragédie de la Nature ». La première est cosmique : la mort du soleil ; la deuxième est spirituelle : la disparition de la conscience de soi ; la troisième est érotique : le viol et la mort de la nymphe. Mais ce tercet représente en même temps une ouverture, il amorce un déploiement qui prendra toute son ampleur dans le second tercet.

$\mathrm{Au}$ deuxième vers du premier tercet, Paz traduit "décor" par rijosa fábula. Rijoso, ici, signifie à la fois querelleur et lubrique. En effet, si l'idée de luxure n'est que sous-entendue dans la version définitive du poème, elle figure expressément dans la première version : "...un or / Néfaste incite pour son beau cadre une rixe / Faite d'un dieu qui croit emporter une nixe ". Paz traduit " décor " par fábula (fable) plutôt que par decorado parce que Mallarmé évoque, dans ce tercet, un épisode de la mythologie nordique (réminiscence, d'après Camille Saula et d'autres critiques, d'une lecture de jeunesse de Heine).

Quant aux licornes "ruant du feu ", cela peut signifier : frappant, donnant de grands " coups de feu " ou ruant, distribuant des ruades de feu (la licorne est un solipède) ou, plus probablement, lançant des flambées avec violence. Gardner Davies, dans son livre Mallarmé et le drame solaire (1959), observe que « ici le verbe ruer a indubitablement le sens de darder ». Mais les licornes ne dardent pas, ne percent pas de flèches; elles donnent des coups de corne, des coups de lance (d'où la traduction par alancear, frapper à coups de lance). L'interprétation est discutable mais elle a le mérite de conserver l'idée de lancer du feu et, corrélativement, celle d'assaut érotique. 
Le premier vers du second tercet consume l'action du premier : la nymphe disparaît dans le miroir. C'est dans les deux vers finals que s'opère le changement : les ailes noires du miroir se ferment sur le corps de la morte et alors, comme le retentissement soudain d'un gong rompant le silence, apparaissent les sept luminaires de la Constellation de la Grande Ourse. Pourquoi la Grande Ourse ? Ici, nous possédons une lettre capitale de Mallarmé à Henri Cazalis qui, préparant un livre : Sonnets et Eaux-fortes, avait demandé au poète sa collaboration. Mallarmé lui envoya la première version du sonnet accompagnée de cette lettre datée de juillet 1868 et où on lit notamment :

C'est confesser qu'il (le poème) est peu "plastique " comme tu me le demandes, mais au moins est-ce aussi «blanc et noir 》 que possible, et il me semble se prêter à une eau-forte pleine de rêve et de vide. Par exemple une fenêtre nocturne ouverte, les deux volets attachés : une chambre avec une personne dedans, malgré l'air stable que présentent les volets attachés, et dans une nuit faite d'absence et d'interrogation, sans meuble, sinon l'ébauche plausible de vagues consoles, un cadre belliqueux et agonisant, du miroir appendu au fond, avec sa réflexion stellaire et incompréhensible, de la Grande Ourse, qui relie au ciel seul ce logis abandonné du monde?

Dans les tercets, le miroir remplit une fonction duelle semblable à celle du coquillage dans les quatrains. Le miroir est, à la fois, un ornement de la chambre et le lieu magique de la métamorphose. Il nous reflète et, en nous enfermant dans sa surface trompeuse, il nous dissout. Instrument de réflexion, le miroir ne nous offre une preuve de notre réalité que pour la démentir aussitôt; il nous dit que nous sommes des images, autrement dit rien. Symbole de la perdition de l'homme et origine de la spéculation, objet d'ornementation et instrument des mages et des sorciers, le miroir reçoit et ensevelit le solstice d'hiver, minuit, la chambre abandonnée, la nymphe morte - tous ces signes qui dénotent la conscience vide - pour ensuite, par une sorte de réversion instantanée, les transformer en scintillements d'étoiles. Le miroir ferme le sonnet et, en même temps, l'ouvre, transmué, sur l'infini : en lui se dissout la conscience personnelle, mais elle renaît en lui comme conscience pure, en accord avec la réalité essentielle du monde. Nous n'avons pas assisté au rite cyclique de la résurrection du soleil, mais à une transmutation d'ordre différent quoique de sens analogue. La constellation n'est pas le soleil, mais son double idéal : sa transfiguration en une grappe de signes, son Idée. Le soleil est un astre en mouvement et les sept étoiles se fixent au fond du miroir dans l'ombre. Le drame de la nature ne se résout pas dans la répétition cyclique et naturelle, mais dans un acte unique. $\mathrm{Si}$ l'acte ne supprime pas le hasard - le soleil pourrait ne pas réapparaître - il l'absorbe et le projette dans une forme immobile, ces sept étoiles qui sont l'idéogramme de la poésie et de la musique. L'acte de transfiguration, dans la conscience vide du poète, est pareil à l'acte que réalise le soleil en apparaissant et en disparaissant à l'horizon, mais, bien que tous deux soient des formes jumelles du hasard, leur signification est différente, opposée mêrme : l'un est cyclique et fatal, l'autre est unique et, d'une certaine façon, libre. Dans le drame naturel, la réalité s'accomplit en un procès qui la décompose et la recompose ; dans l'acte poétique, la réalité s'accomplit en tant qu'Idée : elle cesse d'être processus et se transfigure en signe. Ainsi, aux deux moments naturels de repliement et de déploiernent, en succède un autre, provisoirement définitif : l'apparition de ces étoiles devenues écriture. Le miroir s'est converti en page. Mais ce moment final n'est que provisoire car l'Idée, faite signe, devra maintenant souffrir l'épreuve de la lecture et se réaliser, comme le soleil, dans la mémoire et l'oubli d'un lecteur. Retour à la succession...

Dans sa lettre à Cazalis, Mallarmé écrivait : «...sonnet nu se réfléchissant de toutes les façons ". Sonnet-conque, sonnet-miroir : le dernier vers, lumineux, s'ouvre à la nuit et la musique se résout en silence. Sonnet allégorique de lui-même ${ }^{8}$. 


\section{Notes}

1. "Le paradoxe de la poésie réside en ce qu'elle est, à la fois, universelle et intraduisible. Ce paradoxe se dissipe seulement lorsqu'on considère que si la traduction est effectivement impossible, sa recréation dans une autre langue ne l'est pas. " (Octavio Paz, 1967 : les Manuscrits de Rabindranath Tagore, texte lu à l'Université de Delhi.)

2. Remarquons néanmoins le désir d'assurer une traduction en rimes, dont le schéma est le suivant : A O [IX] A/A A A A/O A O/O O O.

3. Cf., à l'exact opposé symétrique, la vibrante immobilité du « Midi le juste » valérien.

4. Et plus loin : "Aucun dictionnaire, évidemment, ne traduit 'ptyx' par coquillage, sauf cependant le Thesaurus linguae graecae qui donne le sens de repli d'un organe et cite un exemple où 'ptyx' veut dire coquille d'huître. " (L'OEuvre poétique de Stéphane Mallarmé), Paris, Droz, 1940, p. 454.)

5. Remarquons à nouveau l'emploi du présent (capta) par le traducteur, dans le souci d'actualiser la scène.

6. L'atmosphère de cette troisième strophe ne fait-elle pas songer à celle qui règne au début du poème d'Eliot, A Game of Chess (The Waste Land, II)?

7. Le sonnet, finalement, ne figure pas au sommaire du rccueil ćdité par Lemerre. Voir, à ce sujet, Mallarmé, OEuvres complètes, édition critique par H. Mondor et G. Jean-Aubry : 1945, Paris, Gallimard, Bibliothèque de la Pléiade, p. 1489.

8. Titre de la première version du poème (1868). 